\title{
Experimental real-time optimization of a solid oxide fuel cell stack via constraint adaptation
}

\author{
Gene A. Bunin ${ }^{\mathrm{a}}$, Zacharie Wuillemin ${ }^{\mathrm{b}}$, Grégory François ${ }^{\mathrm{a}}$, Arata Nakajo ${ }^{\mathrm{b}}$, Leonidas Tsikonis ${ }^{\mathrm{b}}$, \\ Dominique Bonvin ${ }^{\mathrm{a}, *}$ \\ a Laboratoire d'Automatique, Station 9, Ecole Polytechnique Fédérale de Lausanne, Lausanne 1015, VD, Switzerland \\ ${ }^{\mathrm{b}}$ Laboratoire d'Energétique Industrielle, Station 9, Ecole Polytechnique Fédérale de Lausanne, Lausanne 1015, VD, Switzerland
}

\section{A R T I C L E I N F O}

\section{Article history:}

Received 1 October 2010

Received in revised form

29 March 2011

Accepted 16 April 2011

Available online 12 June 2011

\section{Keywords:}

Applied fuel cell modeling

Constraint adaptation

Optimal fuel cell performance

Real-time optimization

SOFC load tracking

SOFC operation

\begin{abstract}
A B S T R A C T
The experimental validation of a real-time optimization (RTO) strategy for the optimal operation of a solid oxide fuel cell (SOFC) stack is reported in this paper. Unlike many existing studies, the RTO approach presented here utilizes the constraint-adaptation methodology, which assumes that the optimal operating point lies on a set of active constraints and then seeks to satisfy those constraints in practice via the addition of a correction term to each constraint function. These correction terms, also referred to as "modifiers", correspond to the difference between predicted and measured constraint values and are updated at each steady-state iteration, thereby allowing the RTO to iteratively meet the optimal operating conditions of an SOFC stack despite significant plant-model mismatch. The effects of the filter parameters used in the modifier update and of the RTO frequency on the general performance of the algorithm are also investigated.
\end{abstract}

(c) 2011 Elsevier Ltd. All rights reserved.

\section{Introduction}

In the recent decade, fuel cells have received growing attention as viable energy alternatives, and have been advocated as a cleaner and more efficient energy source. However, despite this trend there still remains a number of open problems with fuel cell technology problems that must be resolved before the technology can be put into widespread use and become a practical, capable supplement or substitute for current methods. One of these issues lies in the life of a cell, which may be shortened significantly if the system does not successfully adhere to certain safe operating regions, which may be qualified by constraints on certain manipulated and output variables. In addition to the safety criterion, there is also the criterion of optimality, which states that the cell must be able to operate at the highest efficiency for any immediate power demand. As a direct consequence of these two criteria, the areas of control and optimization have been increasingly called upon for improved fuel cell performance. Unfortunately, despite a large number of theoretical contributions - the majority of which have focused on control $[1-4]$, and only a few on optimization $[5,6]$ - there still remains

\footnotetext{
* Corresponding author. Tel.: +41 216933843; fax: +41 216932574

E-mail address: dominique.bonvin@epfl.ch (D. Bonvin).
}

a significant gap between simulation studies and reported experimental results. To the best of the authors' knowledge, all experimental studies so far have been limited to proton exchange membrane (PEM) cells $[3,4,7]$. In these, many have sought efficiency based on specific criteria that were already known in advance. While this sort of ad hoc approach is certainly sufficient in many cases, it often misses the extra degrees of optimality that could be attained by casting the problem into the traditional optimization framework.

In this paper, a previously developed and simulated constraintadaptation methodology [8] is validated experimentally for a 6-cell solid oxide fuel cell (SOFC) stack. Unlike some previously used methods [5,6], the constraint-adaptation methodology discussed here works on the very simple - yet often true - premise that the optimum (or optimal region) of the problem lies somewhere on the constraints. Therefore, if the proper set of constraints can be met in practice, then the optimality of the process can be largely guaranteed as well. Because of plant-model mismatch, the values of the constrained quantities given by the model will rarely match those provided by the real system, and so an adaptation - carried out by adding a correction term to the modeled constraints - is used to ensure that the constraints used by the optimization match those of the real system. In doing so, the real-time optimization (RTO) iteratively drives the system to the true constraints, with the speed 


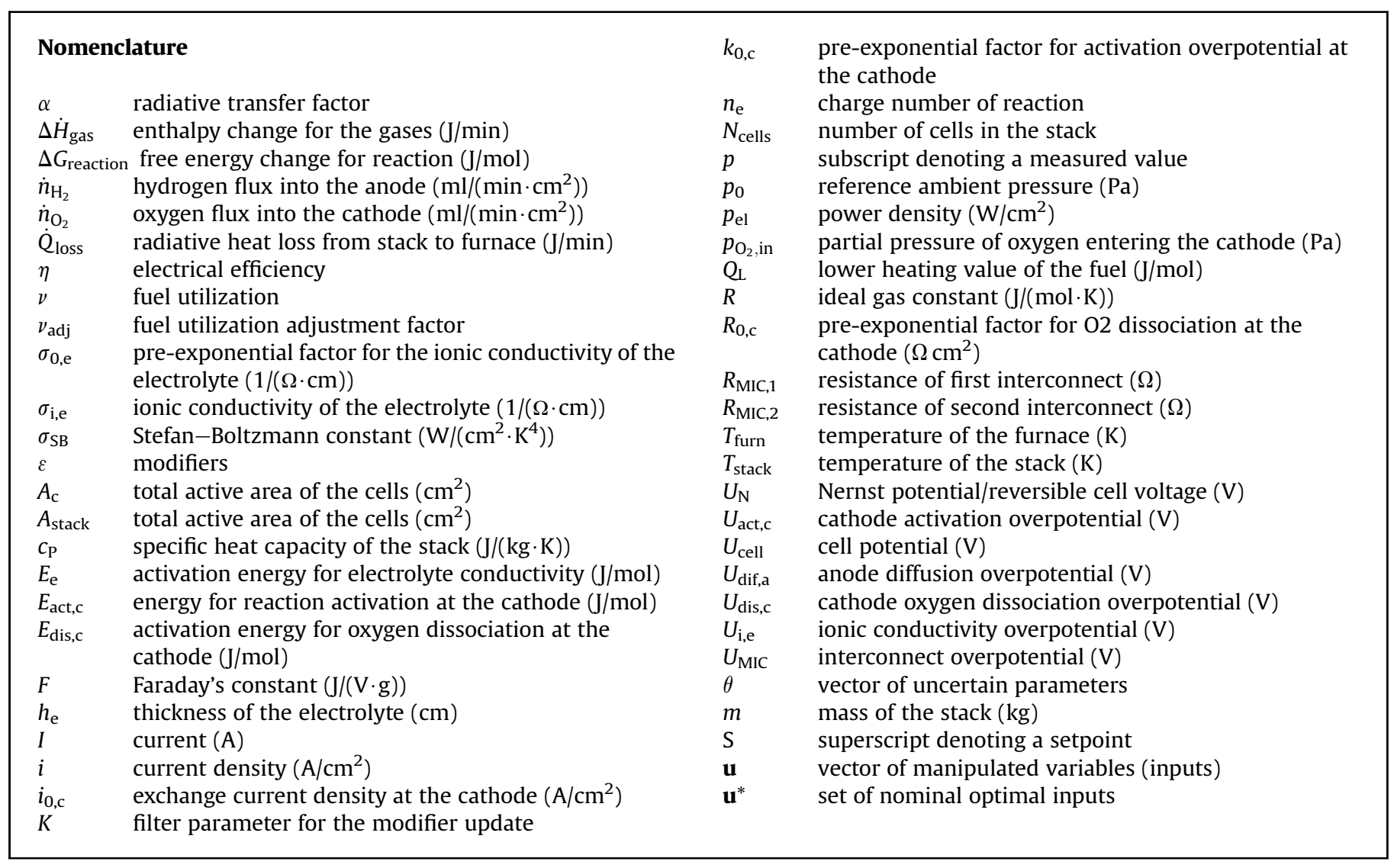

of convergence dictated by the way this correction term is updated. Past studies [9] have demonstrated the robustness of this method to uncertainty, and its ability to reject long-term system degradation.

This paper will be structured as follows. In Section 2, the description of the experimental apparatus and a summary of the model used for the SOFC stack will be given. In Section 3, the constraint-adaptation methodology will be discussed in detail. Its application to the real system will then be outlined and the tested scenarios explained. Section 4 will focus on the results looking not only at the general performance of the experimental stack, but also at the effects of altering the correction term filters and optimization frequency. Section 5 will conclude the paper.

\section{System description}

\subsection{Experimental setup}

The study presented in this article was done on an SOFC shortstack developed at the Industrial Energy Laboratory of the EPFL in Lausanne for HT ceramix-SOFC power $[10,11]$.

The stack consists of planar anode-supported cells with an active area of $50 \mathrm{~cm}^{2}$, pressed between gas-diffusion layers (SOFConnex ${ }^{\mathrm{TM}}$ ) and metallic interconnector plates. The anodes are made of standard nickel/yttrium-stabilized-zirconia (Ni-YSZ) cermet, while the thin electrolyte consists of dense YSZ. The cathodes are made of screenprinted $(\mathrm{La}, \mathrm{Sr})(\mathrm{Co}, \mathrm{Fe}) \mathrm{O}_{3}$, allowing standard operation temperatures between $650{ }^{\circ} \mathrm{C}$ and $850^{\circ} \mathrm{C}$. A detailed description of its construction is available in the literature [10], with a photo of a typical assembly given here in Fig. 1. A stack of 6 cells was used for this study.

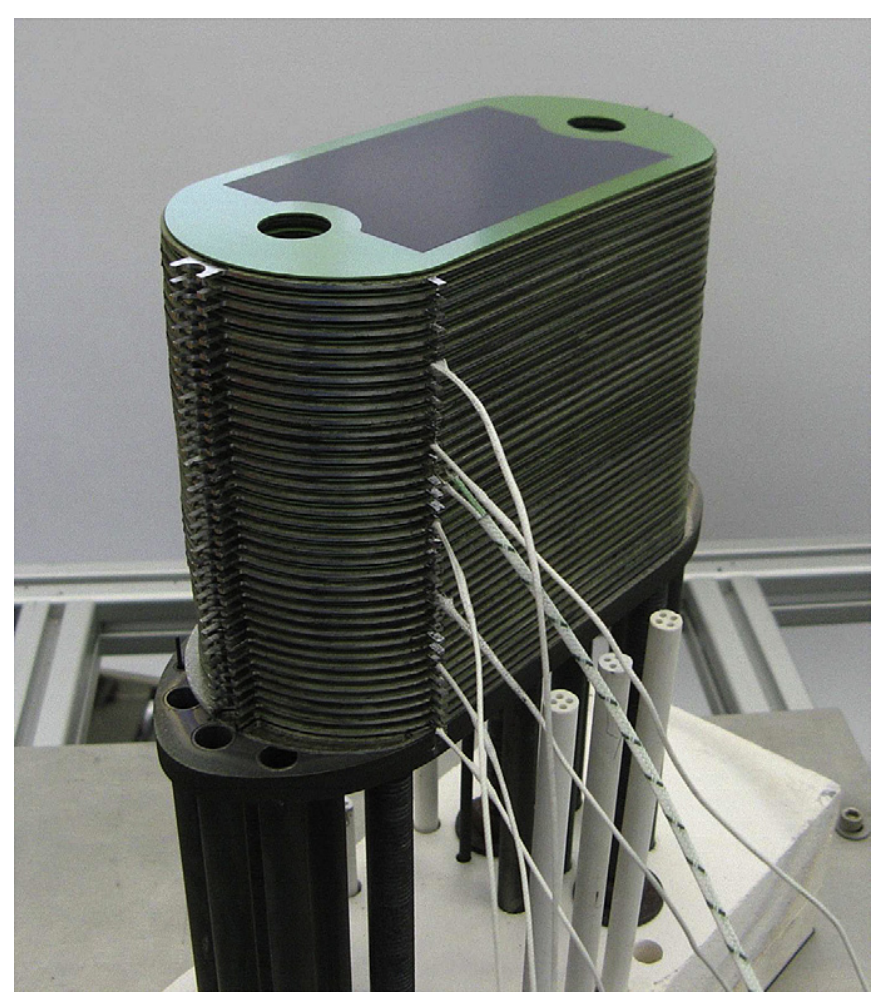

Fig. 1. The setup of a typical stack (Industrial Energy Laboratory of the EPFL in Lausanne). 
The stack was placed in a high-temperature furnace at $775^{\circ} \mathrm{C}$ and connected to a testing station providing controlled flow rates of air and preheated fuel $\left(\sim 770^{\circ} \mathrm{C}\right)$. Additionally, an active load was used to control the delivered current. The control of the testing station was ensured by a LabVIEW interface, in which the RTO algorithm was implemented via a MATLAB Script function.

\subsection{Steady-state SOFC model}

The steady-state model used to optimize the stack is largely similar to that which has been previously reported [8]. As modeling does not comprise a significant contribution in this paper, only the fundamental aspects of the model used are presented here. For a more comprehensive treatment, the interested reader is referred to previous work [8], while the relevant parameter values used in this study are provided in Table 1.

The SOFC is a system fed with $\mathrm{O}_{2}$ (air stream) and $\mathrm{H}_{2}$ (fuel stream), which react electrochemically to produce electrical power and heat. The fuel cells are assembled in stacks in order to reach the desired voltage, and require an exterior load to operate (Fig. 2).

The hydrogen and oxygen fluxes, as well as the current, are the three manipulated variables used to optimize the stack:

$\mathbf{u}=\left[\begin{array}{c}\dot{n}_{\mathrm{H}_{2}} \\ \dot{n}_{\mathrm{O}_{2}} \\ I\end{array}\right]$

As will be shown in the following section, the three outputs of interest are the power density, the cell potential, and the electrical efficiency. The cell potential may be seen as the base component to be modeled, as the other two are direct functions of it. Here, an equivalent-circuit approach is taken [12]:

$U_{\text {cell }}=U_{\mathrm{N}}-U_{\mathrm{act}, \mathrm{c}}-U_{i, e}-U_{\mathrm{dis}, \mathrm{c}}-U_{\mathrm{dif}, \mathrm{a}}-U_{\mathrm{MIC}}$.

The components that make up $U_{\text {cell }}$ are modeled as follows:

$$
\begin{aligned}
& U_{\mathrm{N}}=\frac{-\Delta G_{\text {reaction }}}{n_{\mathrm{e}} F} \\
& U_{\mathrm{act}, \mathrm{c}}=\frac{R T_{\text {stack }}}{F} \sinh ^{-1}\left(\frac{i}{2 i_{0, \mathrm{c}}}\right) \\
& i_{0, \mathrm{c}}=\frac{R T_{\text {stack }}}{F} k_{0, \mathrm{c}} e^{\frac{-E_{\text {act, }}}{R T_{\text {stack }}}}
\end{aligned}
$$

\begin{tabular}{|c|c|c|}
\hline Parameter & Value & Units \\
\hline$\alpha$ & 0.67 & \\
\hline$\nu_{\text {adj }}$ & 0.15 & \\
\hline$\sigma_{0, \mathrm{e}}$ & $1.63 \times 10^{2}$ & $1 /(\Omega \cdot \mathrm{cm})$ \\
\hline$A_{\mathrm{c}}$ & 300 & $\mathrm{~cm}^{2}$ \\
\hline$A_{\text {stack }}$ & 243.47 & $\mathrm{~cm}^{2}$ \\
\hline$E_{\mathrm{e}}$ & $7.622 \times 10^{4}$ & $\mathrm{~J} / \mathrm{mol}$ \\
\hline$E_{\text {act, } \mathrm{c}}$ & $1.533 \times 10^{5}$ & $\mathrm{~J} / \mathrm{mol}$ \\
\hline$E_{\text {dis, c }}$ & $1.489 \times 10^{5}$ & $\mathrm{~J} / \mathrm{mol}$ \\
\hline$h_{\mathrm{e}}$ & $1 \times 10^{-4}$ & $\mathrm{~cm}$ \\
\hline$k_{0, \mathrm{c}}$ & $4.103 \times 10^{7}$ & $1 /\left(\Omega \cdot \mathrm{cm}^{2}\right)$ \\
\hline$p_{0}$ & 101,325 & $\mathrm{~Pa}$ \\
\hline$Q_{\mathrm{L}}$ & $2.7 \times 10^{5}$ & $\mathrm{~J} / \mathrm{mol}$ \\
\hline$R_{0, c}$ & $9.225 \times 10^{-10}$ & $\Omega \cdot \mathrm{cm}^{2}$ \\
\hline$R_{\text {MIC, } 1}$ & $9.872 \times 10^{-3}$ & $\Omega$ \\
\hline$R_{\mathrm{MIC}, 2}$ & $9.872 \times 10^{-3}$ & $\Omega$ \\
\hline
\end{tabular}

Table 1

Nominal model parameter values.

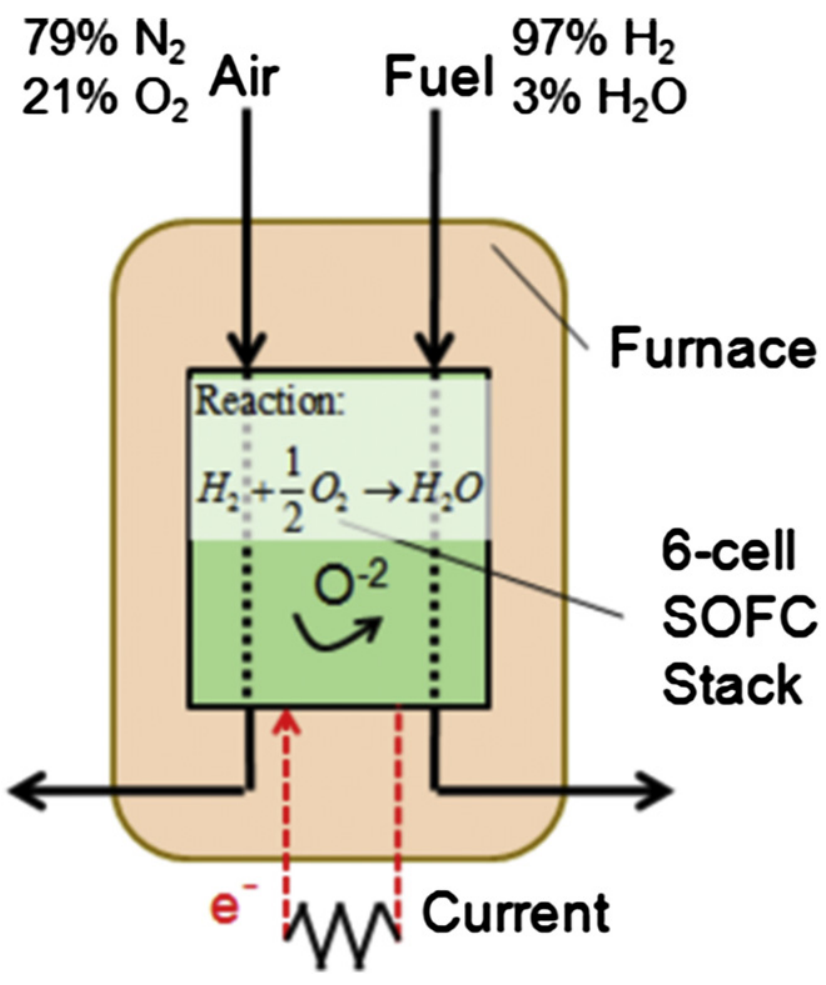

Fig. 2. The diagram of the SOFC system. A fuel feed that is $97 \%$ hydrogen, $3 \%$ water is sent through the anode, while air is fed through the cathode. The stack is polarized with an exogenous current.

$$
\begin{aligned}
U_{\mathrm{i}, \mathrm{e}} & =i \frac{h_{\mathrm{e}}}{\sigma_{\mathrm{i}, \mathrm{e}}} \\
\sigma_{\mathrm{i}, \mathrm{e}} & =\sigma_{0, \mathrm{e}} \mathrm{e}^{\frac{-\mathrm{E}_{\mathrm{e}}}{\mathrm{Rtack}}}
\end{aligned}
$$

$U_{\mathrm{dis}, \mathrm{c}}=R_{0, \mathrm{c}} \sqrt{\frac{p_{0}}{p_{\mathrm{O}_{2}, \text { in }}}} e^{\frac{E_{\mathrm{dis}, \mathrm{c}}}{R T_{\mathrm{stack}}}} i$

$$
\begin{aligned}
U_{\mathrm{dif}, \mathrm{a}} & =-\frac{R T_{\text {stack }}}{2 F} \ln \left[1-\left(\nu+\nu_{\text {adj }}\right)\right] \\
\nu & =\frac{N_{\text {cells }} I}{2 \dot{n}_{\mathrm{H}_{2}} F}
\end{aligned}
$$

$U_{\mathrm{MIC}}=\left(R_{\mathrm{MIC}, 1}+R_{\mathrm{MIC}, 2}\right) I$.

The values of $p_{\mathrm{el}}$ and $\eta$ follow as functions of $U_{\text {cell }}$ :

$$
\begin{gathered}
p_{\mathrm{el}}=\frac{U_{\text {cell }} N_{\text {cells }} I}{A_{\mathrm{c}}} \\
\eta=\frac{p_{\mathrm{el}} A_{\mathrm{c}}}{\dot{n}_{\mathrm{H}_{2}} Q_{\mathrm{L}}} .
\end{gathered}
$$

From Eqs. (2)-(8), it is clear that $U_{\text {cell, }}$ and thus also $p_{\mathrm{el}}$ and $\eta$, depend substantially on the temperature of the stack. A dynamic energy balance is therefore required, and is expressed as:

$$
\begin{aligned}
& m c_{P} \frac{\mathrm{d} T_{\text {stack }}}{\mathrm{d} t}=-\Delta \dot{H}_{\text {gas }}-p_{\mathrm{el}} A_{\mathrm{c}}-\dot{Q}_{\text {loss }} \\
& \dot{Q}_{\text {loss }}=A_{\text {stack }} \alpha \sigma_{\mathrm{SB}}\left(T_{\text {stack }}^{4}-T_{\text {furn }}^{4}\right) .
\end{aligned}
$$

As a means of finding steady-state values for the outputs, the equations in (10) are solved first to obtain the steady-state 
temperature, which is then used to calculate the steady-state values of the potential, power, and efficiency. In general, the response of these quantities to changes in the inputs is practically instantaneous, as most of the response comes on the electrochemical time scale. However, the true steady state of the system is ultimately governed by the temperature as it gradually reaches its new value on the significantly slower thermal time scale [13]. While some SOFC systems may have additional dynamics depending on their setup [14], it is assumed that, for the system at hand, these two time scales - one instantaneous and one on the magnitude of approximately $30 \mathrm{~min}$ - are the only important ones.

In testing this model against the real SOFC stack, one can see a divergence between the predicted potential and the actual value when the current is increased (Fig. 3 illustrates this for one specific set of fluxes, but similar divergence was noted for others). This is particularly crucial for the current range $18-25 \mathrm{~A}$, which is used throughout many of the experiments (Section 4), and which would likely be encountered in application.

\subsection{System constraints}

Two key constraints limit the efficiency in an SOFC. While the cell may deliver a desired electrical power at several different operating conditions (different fuel flows and currents), the maximum electrical efficiency is usually found close to the highest achievable fuel utilization (70-90\%) - defined as the percentage of the fed fuel that reacts. However, to prevent damages to the stack by local fuel starvation and reoxidation of the anode [10], a conservative maximum fuel utilization of $75 \%$ is set. In addition, it is known that significant internal losses occurring at lower cell voltages are detrimental to SOFC stacks. Other phenomena that only occur at lower voltages may also contribute to accelerated cell degradation [10]. For these reasons, a conservative minimum cell voltage of $0.75 \mathrm{~V}$ is set as the second major constraint for the system.

Furthermore, the oxygen-to-hydrogen excess ratio (or simply the "air ratio"), defined as the ratio between the oxygen fed to the system and the oxygen stoichiometrically needed to react with the fuel:

$\lambda_{\text {air }}=2 \frac{\dot{n}_{\mathrm{O}_{2}}}{\dot{n}_{\mathrm{H}_{2}}}$

must be kept within certain bounds so as to avoid steep thermal gradients. For this setup, the ratio is kept between 4 and 7. A lower bound of $3.14 \mathrm{ml} /\left(\mathrm{min} \cdot \mathrm{cm}^{2}\right)^{1}$ is also placed on the fuel feed rate so as to avoid local (or widespread) fuel starvation, and an upper bound of $30 \mathrm{~A}$ is placed on the current to avoid excessive heating [8].

To avoid steep gradients in the stack, limits on the rate of input changes are defined as $0.54 \mathrm{ml} /\left(\mathrm{min}^{2} \cdot \mathrm{cm}^{2}\right), 1.37 \mathrm{ml} /\left(\mathrm{min}^{2} \cdot \mathrm{cm}^{2}\right)$, $2.0 \mathrm{~A} / \mathrm{min}$ for the hydrogen flow, oxygen flow, and current, respectively. Conditional laws are written into the LabVIEW code so that any of these rates can be set to 0 in the case of a prolonged fuel utilization or air ratio violation.

\section{RTO via constraint adaptation}

\subsection{Methodological overview}

Process optimization typically involves the minimization of a cost (or the maximization of a profit) that is subject to certain equality and inequality constraints. This results in a nonlinear

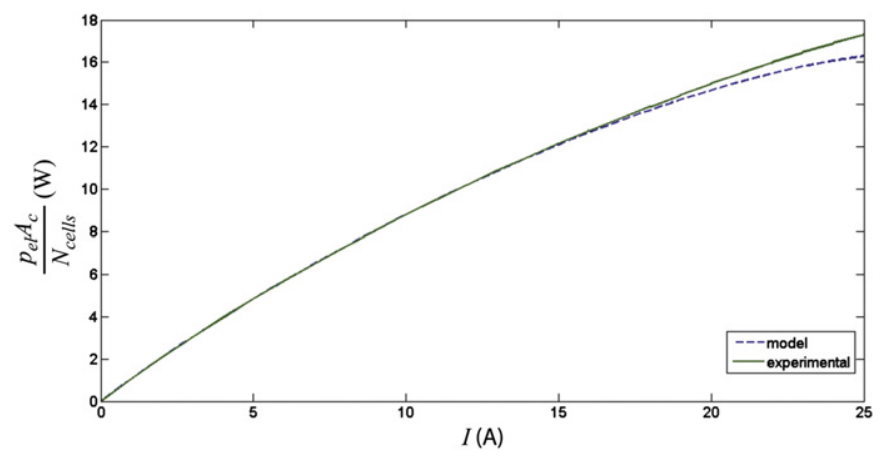

Fig. 3. Power-current (PI) curve for $\dot{n}_{\mathrm{H}_{2}}=5 \mathrm{ml} /\left(\mathrm{min} \cdot \mathrm{cm}^{2}\right), \lambda_{\text {air }}=4$.

programming (NLP) problem which, for the system described in Section 2, may be written in standard optimization form $\mathrm{as}^{2}$ :

$$
\begin{aligned}
\mathbf{u}^{*}=\underset{\mathbf{u}}{\operatorname{argmax}} \cdot & \eta(\mathbf{u}, \theta) \\
\text { s.t. } & p_{\mathrm{el}}(\mathbf{u}, \theta)=p_{\mathrm{el}}^{\mathrm{S}} \\
& U_{\mathrm{cell}}(\mathbf{u}, \theta) \geq 0.75 \mathrm{~V} \\
& \nu(\mathbf{u}) \leq 0.75 \\
& 4 \leq \lambda_{\text {air }}(\mathbf{u}) \leq 7 \\
& u_{1} \geq 3.14 \mathrm{ml} /\left(\mathrm{min} \cdot \mathrm{cm}^{2}\right) \\
& u_{3} \leq 30 \mathrm{~A},
\end{aligned}
$$

with the electrical efficiency $\eta$ acting as the profit function to be maximized, and $p_{\mathrm{el}}^{\mathrm{S}}$ acting as the power demand setpoint to be tracked (this latter effectively gives the optimization a second role as a load-following controller).

Furthermore, we define the following vector of model parameters:

$\theta=\left(E_{\mathrm{act}, \mathrm{c}}, k_{0, \mathrm{c}}, E_{\mathrm{e}}, \sigma_{0, \mathrm{e}}, E_{\mathrm{dis}, \mathrm{c}}, R_{0, \mathrm{c}}, \alpha\right)$.

Essentially, this corresponds to all the key model parameters that are known, but with a certain degree of uncertainty. With the steady-state model described in Section 2.2, the NLP problem (12) may be solved to obtain an optimal set of inputs that theoretically maximizes the cell efficiency while satisfying all of the constraints.

As will be seen, for the system presented in this article, the optimization is actually very intuitive and conforms to the following general rules.

(a) At lower power demands, maximize $\nu$ to maximize efficiency, i.e. $\nu=0.75$ is the active constraint.

(b) For higher power demands, $U_{\text {cell }}=0.75 \mathrm{~V}$ becomes the active constraint, and pushing $\nu$ to 0.75 is no longer optimal as doing so would damage the stack.

Real systems, however, cannot be optimized this way due to modeling errors. Part of this error generally comes from the uncertainty in the parameters $\theta$, while another part comes from the erroneous structure of the model (all real processes are of infinite order and cannot be perfectly modeled even with the perfect parameter estimates). The nominal optimal solution obtained by (12) is thus unlikely to be optimal for the actual SOFC stack.

It should be said that the issue does not appear with regard to the fuel utilization $(\nu)$ constraint. This is because $\nu$ is independent of $\theta$ and its structure is given by definition. As such, it is known with certitude and may be satisfied exactly in (12). However, the same is not true for the cell potential, which cannot be modeled perfectly 
and is often susceptible to uncertainty (and, by consequence, so is the power - as demonstrated in Fig. 3). Therefore, it is possible for the nominal problem to either underestimate or overestimate the $0.75 \mathrm{~V}$ value, resulting in "optimal" inputs that will, in practice, either violate the constraint or reach an early limit by assuming that it is active when it is not.

A common approach to solving problems that deal with this type of uncertainty is to collect the measurements of the real process and to use these to re-identify the original model. In the iterative optimization literature, this is known as the "two-step" approach [15] - the first step consists of solving the identification optimization problem, and the second involves solving the main optimization problem with the updated model parameters. This is an approach that is very intuitive and is thus widely accepted, but which suffers from the fact that estimating the parameters is, in many cases, a nontrivial problem (generally because it is nonconvex and will converge to a local minimum). Potential model inadequacy [16] also makes convergence of this algorithm unlikely from a theoretical point of view.

A simpler alternative, and the one used here, is the resolution of this problem with the use of "modifiers"[8], or correction terms that are added to the uncertain constraint quantities to correct the model values. From a modeling perspective, these are "tailored parameters", as they often don't have any physical significance and are introduced for a specific role - here, they are used to collect all the lumped modeling error and to thereby yield good optimization results, without requiring a potentially difficult identification. Moreover, the convergence of such algorithms to the correct operating point follows from some assumptions that are often acceptable in practice [8]. The qualitative comparison between the two methods is given in Fig. 4.

Applying the modifier methodology to the SOFC problem in (12) requires adding modifiers to the power demand and the cell potential ${ }^{3}$. As the modifiers are generally unable to converge to the optimal values in a single iteration for the nonlinear case (due to varying error at different operating points), convergence is sought over a few iterations, using low-pass filtering [17]. At the $k$ th iteration, the following optimization problem is solved for $\mathbf{u}_{k}$ using the modifiers $\varepsilon_{k-1}^{p_{\text {el }}}$ and $\varepsilon_{k-1}^{U_{\text {cell }}}$ from the previous iteration:

$$
\begin{aligned}
& \mathbf{u}_{k}=\underset{\mathbf{u}}{\operatorname{argmax}} . \eta(\mathbf{u}, \theta) \\
& \text { s.t. } p_{\mathrm{el}}(\mathbf{u}, \theta)+\varepsilon_{k-1}^{p_{\mathrm{el}}}=p_{\mathrm{el}}^{\mathrm{S}} \\
& U_{\text {cell }}(\mathbf{u}, \theta)+\varepsilon_{k-1}^{U_{\text {cell }}} \geq 0.75 \mathrm{~V} \\
& \nu(\mathbf{u}) \leq 0.75 \\
& 4 \leq \lambda_{\text {air }}(\mathbf{u}) \leq 7 \\
& u_{1} \geq 3.14 \mathrm{ml} /\left(\mathrm{min} \cdot \mathrm{cm}^{2}\right) \\
& u_{3} \leq 30 \mathrm{~A} \text {. }
\end{aligned}
$$

Then, the modifiers are updated as follows:

$\varepsilon_{k}^{p_{\mathrm{el}}}=\left(1-K_{p_{\mathrm{el}}}\right) \varepsilon_{k-1}^{p_{\mathrm{el}}}+K_{p_{\mathrm{el}}}\left[p_{\mathrm{el}, \mathrm{p}}\left(\mathbf{u}_{k}\right)-p_{\mathrm{el}}\left(\mathbf{u}_{k}, \theta\right)\right]$

$\varepsilon_{k}^{U_{\text {cell }}}=\left(1-K_{U_{\text {cell }}}\right) \varepsilon_{k-1}^{U_{\text {cell }}}+K_{U_{\text {cell }}}\left[U_{\text {cell }, \mathrm{p}}\left(\mathbf{u}_{k}\right)-U_{\text {cell }}\left(\mathbf{u}_{k}, \theta\right)\right]$

At the optimum (for $k \rightarrow \infty$ ), the modifiers will have converged and will simply be the error between the actual and estimated values:

\footnotetext{
${ }^{3}$ Note that, in principle, a modifier should be added to the efficiency as well, since it is prone to parametric (and structural) uncertainty. However, from an optimization perspective, the addition of a correction term to the cost does not affect the solution, and so this addition is unnecessary and is thus omitted.
}

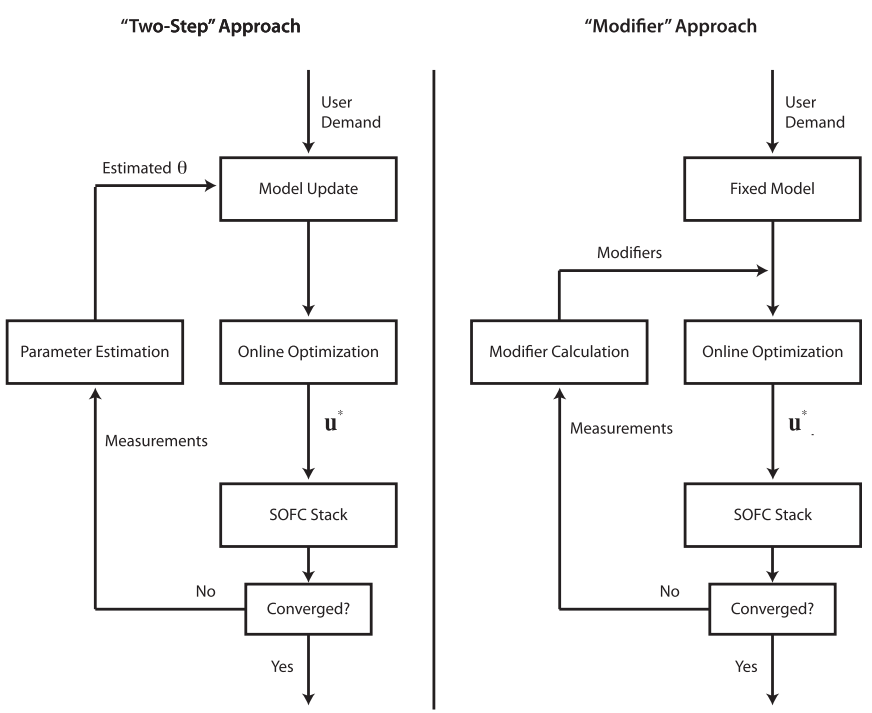

Fig. 4. Qualitative comparison of the standard "two-step" algorithm with the modifieradaptation algorithm used in this work. The main difference lies in how the main optimization problem is updated to account for measured model errors. In the twostep approach, the parameters of the original model are re-estimated at every iteration, resulting in a parameter estimation problem that may not be tractable or that may yield poor results. For the modifier-adaptation scheme, the original model is left alone, and the parameter estimation is replaced by a simple calculation of the modifiers.

$\varepsilon_{\infty}^{p_{\text {el }}}=p_{\text {el,p }}\left(\mathbf{u}_{\infty}\right)-p_{\text {el }}\left(\mathbf{u}_{\infty}, \boldsymbol{\theta}\right)$
$\varepsilon_{\infty}^{U_{\text {cell }}}=U_{\text {cell }, \mathrm{p}}\left(\mathbf{u}_{\infty}\right)-U_{\text {cell }}\left(\mathbf{u}_{\infty}, \boldsymbol{\theta}\right)$.

With the addition of the modifiers, the solution given by the optimization is guaranteed, upon convergence, to satisfy the constraints of the plant [8]. The general algorithm proceeds as follows:

(1) Set $k:=1$ and choose initial values for the modifiers $\varepsilon_{0}^{p_{\text {el }}}$ and $\varepsilon_{0}^{U_{\text {cell }}}$.

(2) Solve the modified optimization problem (14) to obtain new input values $\mathbf{u}_{k}$.

(3) Apply these input values and let the system converge to a new steady state.

(4) Update the modifiers according to (15). If $\left\|\mathbf{u}_{k}-\mathbf{u}_{k-1}\right\| \leq \delta$ (where $\delta$ is a user-specified threshold), assume convergence. If not, set $k:=k+1$ and return to Step 2 .

The block diagram of the algorithm is presented in Fig. 5.

\subsection{Application to the real stack}

To test the effectiveness of the proposed methodology, a preset power demand profile,

$p_{\mathrm{el}}^{\mathrm{S}}(t)= \begin{cases}0.30 \mathrm{~W} / \mathrm{cm}^{2} & t \leq 90 \mathrm{~min} \\ 0.38 \mathrm{~W} / \mathrm{cm}^{2} & 90 \mathrm{~min}<t \leq 180 \mathrm{~min} \\ 0.30 \mathrm{~W} / \mathrm{cm}^{2} & t>180 \mathrm{~min}\end{cases}$

is chosen to demonstrate how the change in active constraints (from $\nu$ to $U_{\text {cell }}$ ) may occur. Note that the power demand profile $p_{\mathrm{el}}^{\mathrm{S}}(t)$ acts as a disturbance: in other words, it is not known in advance when and how the power demand may change in application. An RTO frequency of one iteration per $30 \mathrm{~min}$ is used, as this is the time needed for the actual system to reach steady state. 


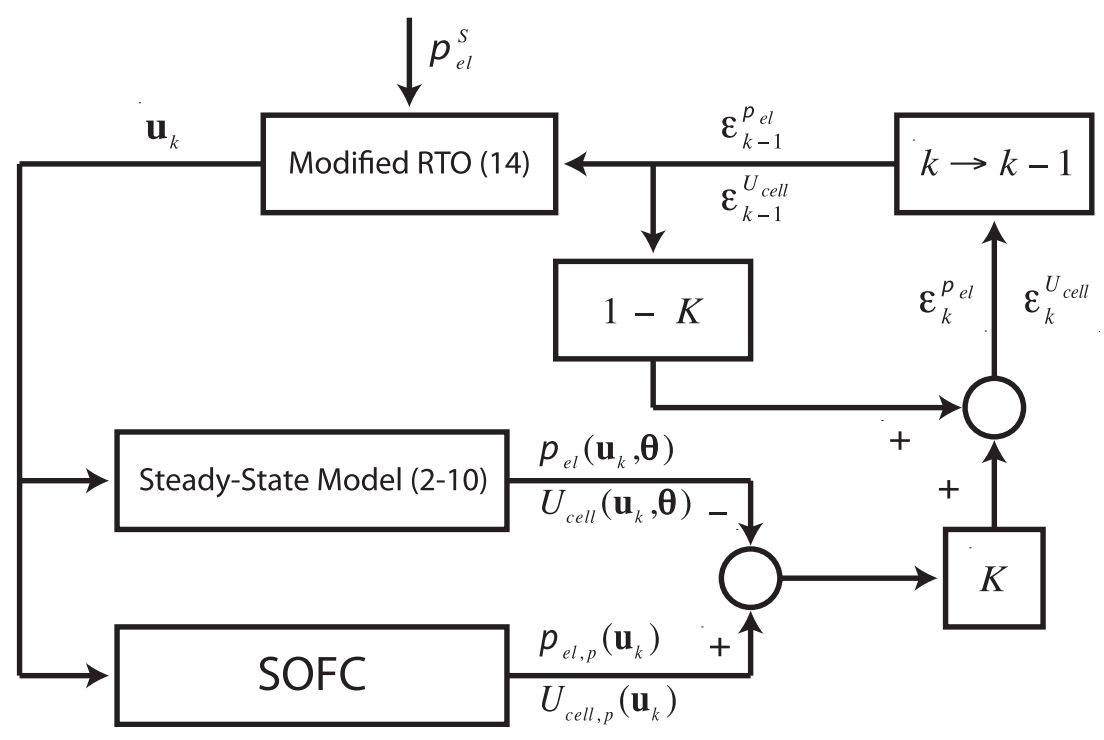

Fig. 5. Constraint-adaptation RTO scheme.

The initial (sub-optimal) steady-state inputs are $5 \mathrm{ml} /\left(\mathrm{min} \cdot \mathrm{cm}^{2}\right)$, $12.77 \mathrm{ml} /\left(\mathrm{min} \cdot \mathrm{cm}^{2}\right)$, and $20 \mathrm{~A}$ for the fuel flux, oxygen flux, and current, respectively.

\section{Results and discussion}

\subsection{Optimal power tracking with different filters}

The scenario described in Section 3.2 is tested in the SOFC system for different values of the filter parameters. For this set of scenarios, $K_{U_{\text {cell }}}$ is set equal to $K_{p_{\mathrm{el}}}$, and the two are varied together. Three different filter values of $0.4,0.7$, and 1.0 (the latter corresponding to full adaptation, i.e. no filtering) are investigated. The results are presented in Figs. $6-8$.

It is observed that the optimizer immediately seeks to maximize the air ratio for all cases (note the relative values of the hydrogen and oxygen fluxes). This is because, without any parasitic losses to the air blower in the objective function (which may be accounted for in simulations [8]), there is no reason for the optimizer to keep it at low levels. The fuel utilization also seems extremely sensitive to small disturbances in the hydrogen flux, which leads to occasional fluctuations and violations in this constraint. Finally, there is a "dip" in the fuel utilization during power changes, which is due to the fact that the system must keep the air ratio below its upper limit during the transient and, for this reason, does not decrease the hydrogen flux quickly enough to match a decrease in current. What results is a temporarily low fuel utilization.

Otherwise, as expected, the filter parameters affect the speed of convergence to the optimum. With a low filter gain, as in Fig. 6, convergence is slow and damped, and a significant offset between the desired value and what is actually observed persists even after two iterations. For the medium-sized filter gain in Fig. 7, it is quicker but still damped. For the full adaptation case in Fig. 8, convergence is fast but oscillatory, as the errors between the modeled power and real power switch signs during convergence. In some cases, it may be possible that the oscillatory behavior noted for the no-filtering case would not converge, thus leading to divergence and instability. In such scenarios, a more careful design of the filter is needed [8,17], but this is not the case here.

of great interest is the way the algorithm handles the constraints. There are no algorithmic issues for fuel utilization, as there is no uncertainty in the model. Noise in the lower-layer controllers does appear to be an issue, as sporadic violations occur during the tracking of the constraint. This is, however, a likely flaw of the control system rather than of the optimization algorithm.
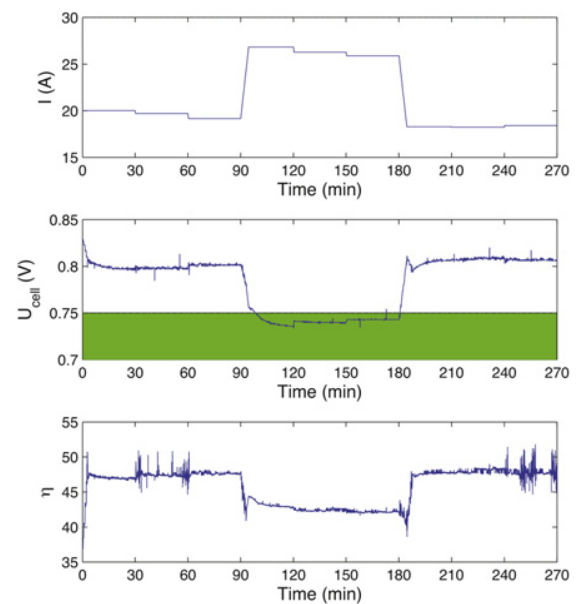

Fig. 6. RTO performance with $K_{p_{\mathrm{el}}}=K_{U_{\text {cell }}}=0.4$. 

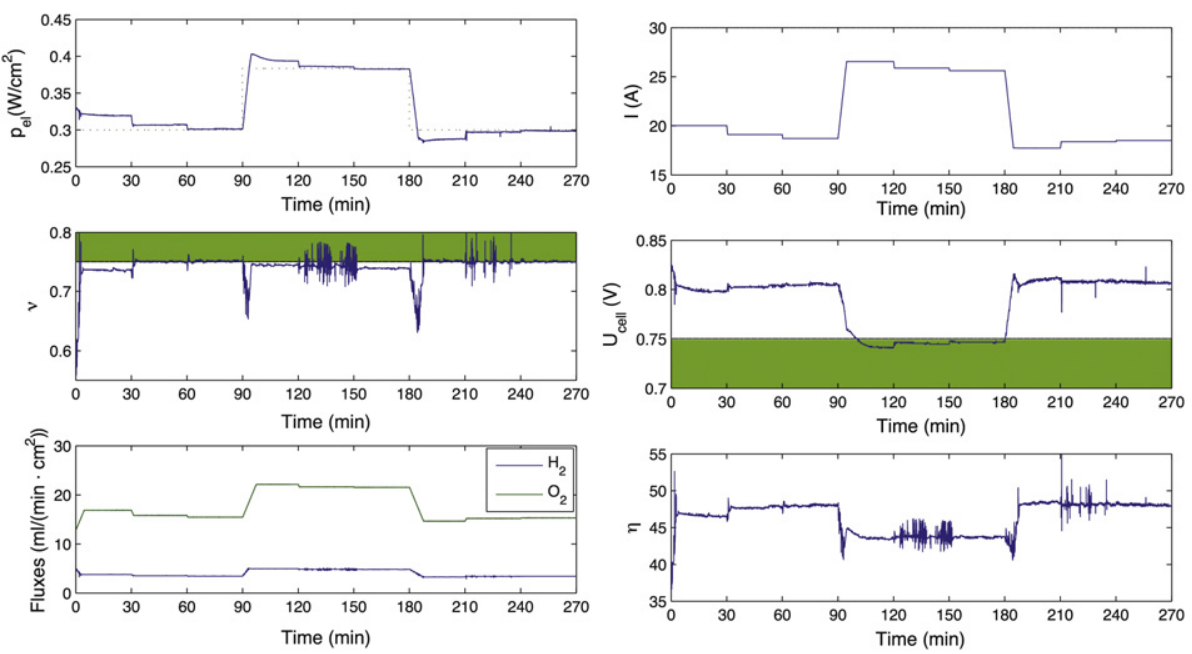

Fig. 7. RTO performance with $K_{p_{\text {el }}}=K_{U_{\text {cell }}}=0.7$.

Algorithmic issues do occur with the constraint on the cell potential, as it is initially violated when the algorithm tries to use the modifiers obtained for a low power demand to compute the optimum for a higher one. Subsequent iterations bring this closer to the appropriate value, but the initial violation proves problematic. With the steady-state RTO alone, there seems to be no ready means to solve this problem without resorting to conservative constraint backoffs, as the converged modifiers from the low power level always lead to this sort of violation in the higher one.

Better performance and faster convergence may be achieved by tuning the filters in an ad hoc manner (see Fig. 9). However, this does not provide a systematic solution to the problem of constraint violations. In the next section, the use of RTO at high frequency is proposed, and it is shown that this method may successfully resolve the aforementioned problems.

\subsection{Fast RTO}

Although SOFCs with the capability to track constant power demand profiles may be of great use, many applications involve power demand changes that occur much more frequently than on the scale proposed in the preceding experiments. For this reason, the use of "fast" RTO is also investigated. Instead of waiting for the system to reach the true steady state with constant temperature ( $\sim 30 \mathrm{~min}$ ) before implementing the RTO, it is assumed that most of the output response takes place at the electrochemical scale $(<1 \mathrm{~s})$. With this assumption, the temperature dynamics can be ignored and treated like a slow-scale parametric drift, and the RTO frequency is increased to an action every $10 \mathrm{~s}$. Filter values of $K_{p_{\mathrm{el}}}=$ 0.85 and $K_{U_{\text {cell }}}=1.0$ are used. A power-demand profile spanning $1 \mathrm{~h}$ of operation is generated, with a new random power demand between 0.30 and $0.38 \mathrm{~W} / \mathrm{cm}^{2}$ being specified every $5 \mathrm{~min}$. Towards the end of the experiment, a 15 min stretch is used to manually test the ability of this algorithm to meet the maximum power without violating the constraint. A converged system at $p_{\mathrm{el}}^{S}=0.30 \mathrm{~W} / \mathrm{cm}^{2}$ is used as a starting point. The results are presented in Fig. 10.

The outcome is very promising. Owing to the fact that there is a very large difference between the two time scales, the optimizer does not suffer from the lack of knowledge of the true steady-state bias and is able to also act as a very effective controller - quickly tracking the appropriate power demand without needing any extensive tuning. Via its role as an optimizer, it maintains the efficiency at near-optimal levels throughout the course of
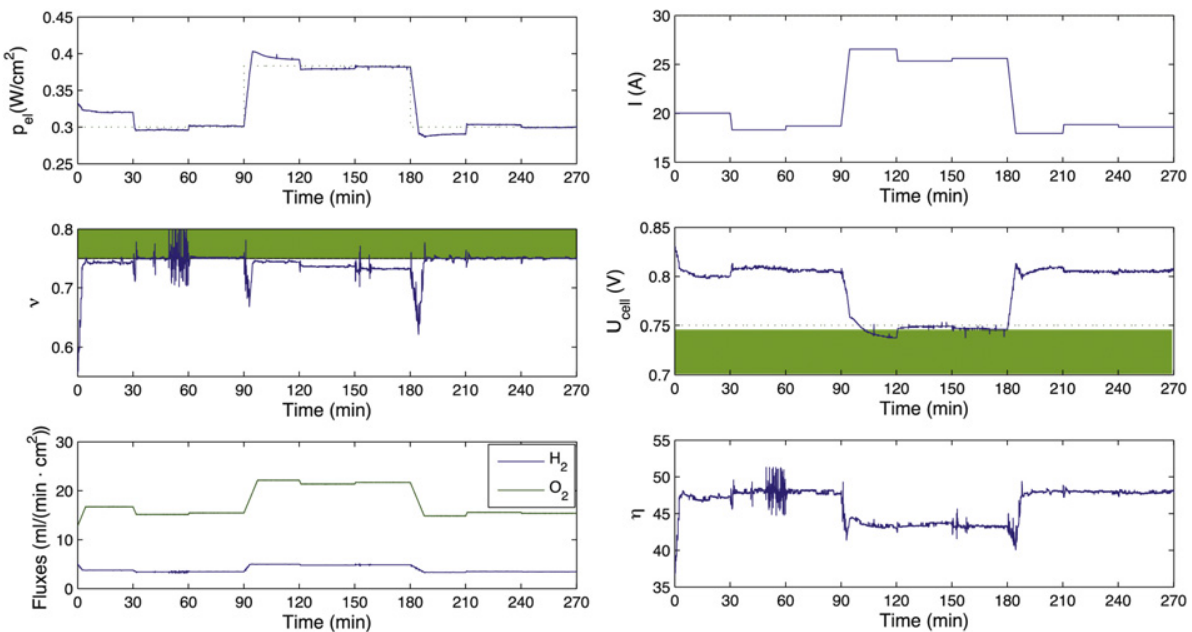

Fig. 8. RTO performance with $K_{p_{\mathrm{el}}}=K_{U_{\text {cell }}}=1.0$. 

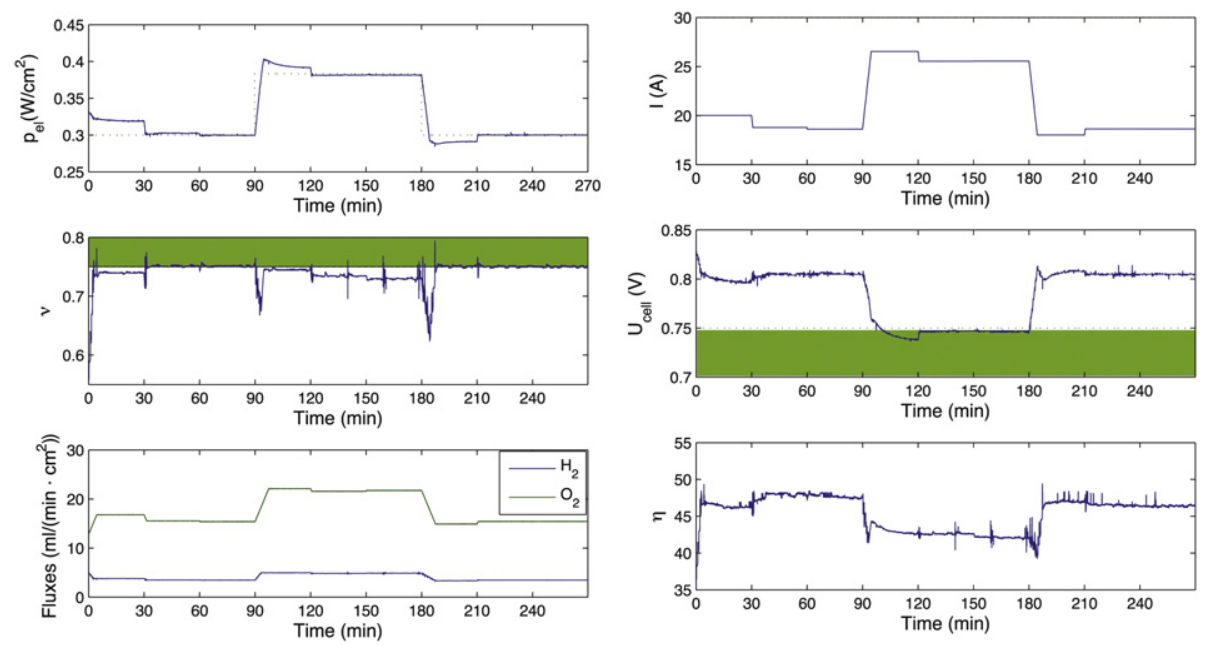

Fig. 9. RTO performance with $K_{p_{\mathrm{el}}}=0.85$ and $K_{U_{\text {cell }}}=1.0$.
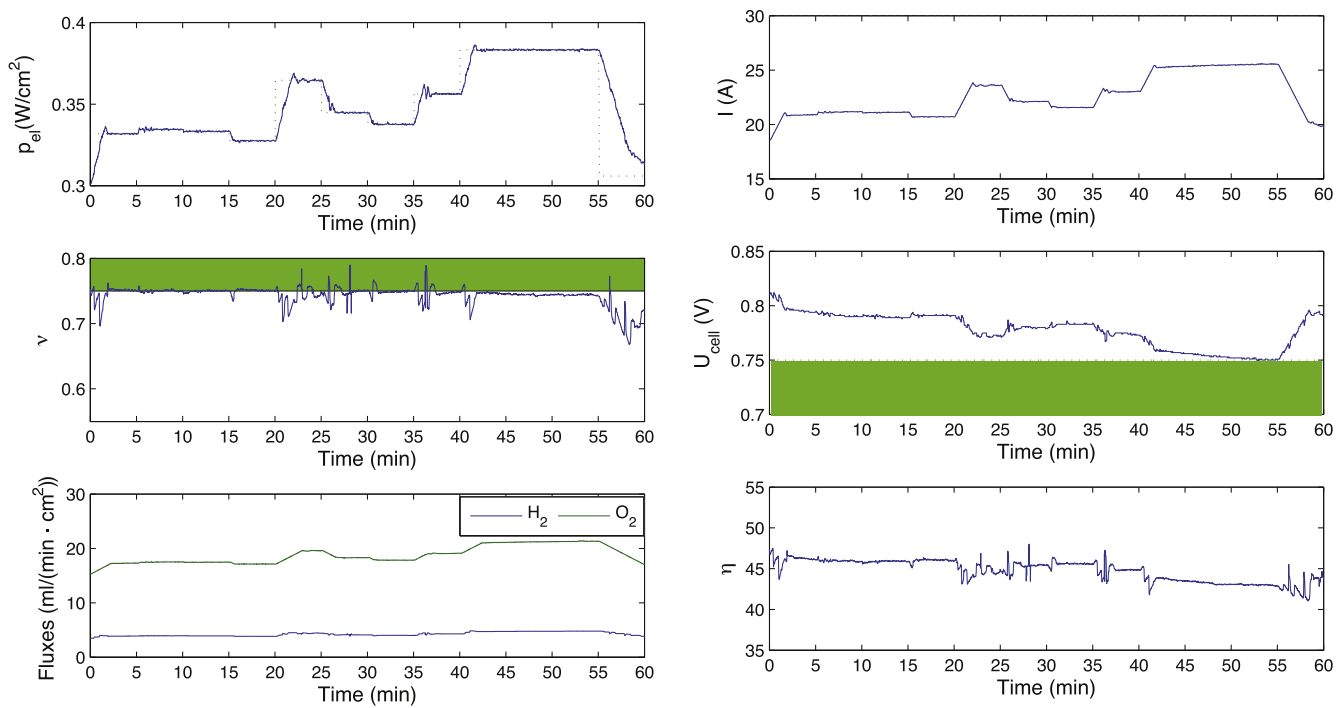

Fig. 10. Performance of the fast RTO.

operation. Finally, unlike in the previous cases where the slow updates allowed prolonged violations, the potential constraint is approached and met, rather than violated, with this method. One does notice, however, that new power demands cannot be met if the change in the demand is too large (the last iteration in Fig. 10), but this is again a limitation of the physical system at hand, rather than that of the algorithm.

\section{Conclusions}

An RTO with constraint adaptation has been investigated for an experimental SOFC stack. It is shown that, despite significant plantmodel mismatch, the adaptive optimization algorithm is able to successfully drive the SOFC stack to its true optimal region, converging to the specified power demand and to the proper active constraint. Additional studies demonstrate the effects of the filter values on the convergence rates. A high-frequency RTO is also attempted, and it is shown that ignoring the transient effects of the temperature does not harm the performance of the algorithm. As a result, the RTO acts as both an optimizer and a controller in this case, and is able to converge quickly and safely to the optimum.
Though not addressed in this paper, this mechanism still has open issues that must be looked at. A more rigorous theoretical treatment of the filter tuning is still needed. Furthermore, it is also important to note that the high frequency RTO approach used in this study may not be possible for more complicated systems, where higher-dimensional input spaces and increased likelihood of local extrema in the model structure may make converging to a good optimum too time-consuming to be implemented on such a fast scale. In these cases, other approaches may be needed to guarantee safe convergence. As such, the efficacy of the proposed method for more complicated SOFC problems, such as those involving steam reformers, cost criteria with parasitic losses, or heat-demand following with co-generation, is a topic of great interest that is yet to be studied.

\section{Acknowledgments}

The authors would like to thank Stefan Diethelm and Luis Quina, from the Industrial Energy Laboratory of the EPFL in Lausanne, for their assembly of the stack, and for all subsequent laboratory assistance. 


\section{References}

[1] Gaynor R, Mueller F, Jabbari F, Brouwer J. On control concepts to prevent fuel starvation in solid oxide fuel cells. J Power Sources 2008;180:330-42.

[2] Zhang X, Chan S, Ho H, Li J, Li G, Feng Z. Nonlinear MPC based on the moving horizon state estimation for the solid oxide fuel cell. Int J Hydrogen Energy 2008;33:2355-66.

[3] Arce A, del Real A, Bordons C, Ramírez D. Real-time implementation of a constrained MPC for efficient airflow control in a PEM fuel cell. IEEE Trans Ind Electr 2010;57(6):1892-905.

[4] Yang Y, Wang F, Chang H, Ma Y, Weng B. Low power proton exchange membrane fuel cell system identification and adaptive control. J Power Sources 2007; 164:761-71.

[5] Golbert J, Lewin D. Model-based control of fuel cells: (2) optimal efficiency. J Power Sources 2007;173:298-309.

[6] Zhong ZD, Huo HB, Zhu XJ, Cao GY, Ren Y. Adaptive maximum power point tracking control of fuel cell power plants. J Power Sources 2008; 176:259-69.

[7] Rodatz P, Paganelli G, Sciarretta A, Guzzella L. Optimal power management of an experimental fuel cell/supercapacitor-powered hybrid vehicle. Control Eng Practice 2005;13:41-53.

[8] Marchetti A, Gopalakrishnan A, Chachuat B, Bonvin D, Tsikonis L, Nakajo A Wuillemin Z, Van herle J. Real-time optimization of a solid oxide fuel cell stack. J Fuel Cell Sci Tech, in press, doi: 10.1115/1.4003976.
[9] Bunin G, François G, Bonvin D. Two-layered real-time optimization of a solid oxide fuel cell stack. In: Kothare M, Tade M, Wouwer A, Smets I, editors. Ninth international symposium on dynamics and control of process systems; 2010. p. 839-44.

[10] Wuillemin Z. Experimental and modeling investigations on local performance and local degradation in SOFC (no. 4525). Ph.D. thesis, EPFL; 2009. http:// library.epfl.ch/theses/?nr=4525.

[11] Diethelm S, Van Herle J, Wuillemin Z, Nakajo A, Autissier N, Molinelli M. Impact of materials and design on solid oxide fuel cell stack operation. J Fuel Cell Sci Technol 2008;5(3):031003.1-6.

[12] Nakajo A, Wuillemin Z, Van Herle J, Favrat D. Simulation of thermal stresses in anode-supported solid oxide fuel cell stacks. Part I: probability of failure of the cells. J of Power Sources 2009;193(1):203-15.

[13] Achenbach E. Response of a solid oxide fuel cell to load change. J Power Sources 1995;57:105-9.

[14] Stiller C, Thorud B, Bolland O, Kandepu R, Imsland L. Control strategy for a solid oxide fuel cell and gas turbine hybrid system. J Power Sources 2006;158:303-15.

[15] Chen C, Joseph B. On-line optimization using a two-phase approach: an application study. Ind Eng Chem Res 1987;26:1924-30.

[16] Forbes J, Marlin T. Design cost: a systematic approach to technology selection for model-based real-time optimization systems. Comput Chem Eng 1996;20: 717-34.

[17] Chachuat B, Marchetti A, Bonvin D. Process optimization via constraints adaptation. J Process Contr 2008;18:244-57. 